\title{
Distorted vacuum black holes in the canonical ensemble
}

\author{
O. B. Zaslavskii \\ Department of Physics and Technology, \\ Kharkov V.N. Karazin National University, \\ 4 Svoboda Square, Kharkov 61022, Ukraine and \\ Institute of Mathematics and Mechanics, Kazan Federal University, \\ 18 Kremlyovskaya St., Kazan 420008, Russid*
}

\begin{abstract}
We consider a vacuum static spacetime in a finite size cavity. On the boundary, we specify a metric and a finite constant local temperature $T$. No spherical or any other spatial symmetry is assumed. We show that (i) inside a cavity, only a black hole or flat spacetime are possible, whereas a curved horizonless regular space-time is excluded, (ii) in the limit when the horizon area shrinks, the Hawking temperature diverges, (iii) for the existence of a black hole, $T$ should be high enough. When $T \rightarrow \infty$, a black hole phase is favorable thermodynamically. Our consideration essentially uses the coordinate system introduced by Israel in his famous proof of the uniqueness theorem.

PACS numbers: 04.70.Bw, 97.60.Lf
\end{abstract}

\section{INTRODUCTION}

For consistent thermodynamics of self-gravitating systems, a finite size is a key point to be taken into account. This was demonstrated first by York, Jr. [1] who showed that account for the boundary resolves the problems that seem to prevent the construction of the canonical ensemble for Schwarzschild black holes and leads to new interesting features. In particular, it turned out that for a given temperature $T$ on the boundary and the radius $R$ of the cavity, there exist two branches of black hole metrics, if $T$ is high enough, one of two branches being locally or even globally stable. This also showed that in discussing thermal

*Electronic address: zaslav@ukr.net 
nucleation of black holes from hot empty space-time [2], one cannot neglect the presence of the boundary without which the thermal ensemble does not exist at all.

Further, some general formulas describing thermal properties of the gravitating ensembles were derived [3] - [6]. Unfortunately, application of the general formalism in concrete analysis was done for spherically symmetric metrics only [7], [8] since the explicit expression for distorted black holes is, as a rule, absent or too complicated. This somewhat slowed down further progress in this area.

The aim of the present note is to show that there are some simple but useful properties of distorted black holes in the canonical ensemble that can be inferred with minimum information, almost "from nothing". It turned out that this step can be performed due to using a simple and elegant coordinate frame used by W. Israel [9] in proving his famous uniqueness theorems for black holes. Now, however, one should bear in mind that in contrast to [9], our space-time is not asymptotically flat due to the presence of the boundary and this is the crucial point.

\section{BASIC EQUATIONS}

In the coordinate frame of Ref. [9], the metric can be written in the form

$$
d s^{2}=-V^{2} d t^{2}+\rho^{2} d V^{2}+\gamma_{a b} d x^{a} d x^{b} .
$$

Here, $a, b=1,2$. It is supposed that the metric coefficients do not depend on $t$. It is seen from (1) that

$$
\rho^{-2}=(\nabla V)^{2} .
$$

In the horizon limit,

$$
\rho \rightarrow \rho_{H}=\frac{1}{\kappa},
$$

where $\kappa$ is the surface gravity (see, e.g.. eqs. $105-107$ in [10]).

Then, the Hawking temperature

$$
T_{H}=\frac{\kappa}{2 \pi}=\frac{1}{2 \pi \rho_{H}} .
$$

Eq. (32) of [9] gives us

$$
\frac{\partial}{\partial V}\left(\frac{\sqrt{\gamma}}{\rho}\right)=0
$$


whence

$$
\rho=\sqrt{\gamma} C\left(x^{1}, x^{2}\right)
$$

Eq. (39) of [9] tells us that the Kretschmann scalar $K r$ in the vacuum space-time

$$
\frac{K r}{8}=\frac{1}{8} R_{A B C D} R^{A B C D}=\left(V \rho^{)^{-2}}\left[K_{a b} K^{a b}+2 \rho^{-2} \rho_{; a ; b} \rho^{a ; b}+\rho^{-4}\left(\frac{\partial \rho}{\partial V}\right)^{2}\right] .\right.
$$

\section{PROPERTIES OF THE CANONICAL ENSEMBLE}

Usually, when dealing with thermodynamic description of some space-time, one first finds its metric (or takes the already known one) and only afterwards ascribes thermodynamic parameters to the system. Meanwhile, a coherent approach to finite size thermodynamic implies something quite different. What is done in the problem are boundary data that are specified on some surface that is not necessarily spherical. These includes the boundary metric $\gamma_{a b}$ and the local temperature. Also, for specifying a solution of field equations, we need $\rho$ and $K_{a b}$. Integrating equations of motion in the inward direction, one can in principle (but not in practice) find these solutions inside a cavity. Meanwhile, for our purpose we need much less information (see below) that require only one equation plus regularity conditions.

Statement 1 . If inside a cavity the vacuum space-time is not flat, it cannot be horizonless.

Proof. If the space-time is flat, $V \equiv 1$ and cannot be taken as an independent variable. We assume that it is not flat, so the metric in the form (1) can be used. Let us suppose that there is no horizon, so the metric has a regular centre. This means that for some $V=V_{1}>0$ the quantity $\sqrt{\gamma}=0$. Then, according to (6),$\rho \rightarrow 0$ as well when $V \rightarrow V_{1}$. In (17) this entails that the finiteness of $K r$ requires

$$
\frac{\partial \rho}{\partial V} \sim \rho^{3}, \frac{1}{\rho^{2}} \sim V+\text { const }, \rho\left(V_{1}\right) \neq 0 .
$$

This is in contradiction with (6).

Thus there are only two phases take part in thermodynamic competition: the flat spacetime and a black hole.

Statement 2. A vacuum black hole with a finite horizon area cannot be extremal $(\kappa \neq 0)$.

Proof. If $\kappa=0$, it follows from (3) that $\rho_{H}=\infty$. Then, it follows from (66) that $\gamma_{H}=\infty$ as well in contradiction with the assumption. 
Statement 3. If the horizon area shrinks, the Hawking temperature in this limit grows unbounded:

$$
\lim _{A_{H} \rightarrow 0} T_{H}=\infty
$$

Proof. The area $A$ of any equipotential surface $V=V_{1}=$ const is equal to

$$
A=\int d x^{1} d x^{2} \sqrt{\gamma}
$$

Here, $\gamma=\gamma\left(V, x^{1}, x^{2}\right)$, integration is performed in some fixed intervals $a_{1} \leq x^{1} \leq b_{1}$, $a_{2} \leq x^{2} \leq b_{2}$ (for instance, one can choose the analogue of angular variables). Therefore, the condition that $A \rightarrow 0$ entails that $\sqrt{\gamma} \rightarrow 0$ as well. Then, it follows from (6) that $\rho_{H} \rightarrow 0$, so that $T_{H} \rightarrow \infty$ according to (4). Eq. (9) generalizes the corresponding property of the Schwarzschild metric, where $T_{H}=\left(4 \pi r_{+}\right)^{-1}, r_{+}$being the horizon radius.

It is essential that we deal with equipotential surfaces. For comparison let us consider, say, the Schwarzschild metric. We can take an arbitrary point and encircle it by a small sphere with minimum and maximum value of the standard Schwarzschild coordinate $r_{1}$ and $r_{2}$. Obviously, such a sphere is not equipotential surface and the above reasonings do not apply. When $r_{2} \rightarrow r_{1}$, the area vanishes although $\sqrt{\gamma}=r^{2} \sin ^{2} \theta$ remains separated from zero.

Statement 4. Black hole solutions are possible only in the high temperature phase, $T>T_{m}$, where the concrete value $T_{m}$ is determined by the boundary conditions (and thus cannot be found in a general form).

Proof. Let $\beta \equiv T^{-1}$ be the inverse temperature on the boundary. It follows from the Tolman formula that

$$
\beta=\beta_{0} V_{B}
$$

where $\beta_{0}=T_{H}^{-1}$ is a constant, $V_{B}$ is the value of $V$ on the boundary.

For a black hole, the horizon area $A_{H}$ lies in the interval $0<A_{H}<A_{B}$, where $A_{B}$ corresponds to the boundary. It follows from (9) that in the limit when the horizon shrinks to the point, so $A_{H} \rightarrow 0$, the quantity $\beta \rightarrow 0$ due to the factor $\beta_{0}$. On the other hand, if a black hole occupies almost the whole cavity, the boundary almost coincides with the horizon, so $V_{B}$ approaches $V$ on the horizon where it is equal to zero. Now, $\beta \rightarrow 0$ due to the second factor in (11). Thus the quantity $\beta$ vanishes in both limits: for the minimum possible area (equal to zero) and the maximum one (corresponding to the boundary). Therefore, in some 
intermediate point $A_{H}=A_{m}$ the inverse temperature $\beta$ should pass through the maximum point equal to some $\beta_{m}=\beta\left(A_{m}\right)$. This proves the statement. For extremal black holes it would be possible for $\beta$ to diverge but we deal now with nonextremal ones.

Statement 5 . For a given $T$, there exist at least two branches of black holes, one of which (at least in some interval of temperatures) is locally stable.

Proof. As $\beta(A)$ has two zeros at $A_{H}=0$ and $A_{H}=A_{B}$, the branch $A_{m}<A_{H}<A_{B}$ has $\frac{d \beta}{d A_{H}}<0$, if it is monotonic. Then, $\frac{d T}{d A_{H}}>0$. Meanwhile, the heat capacity $C=\frac{d E}{d T}=$ $T \frac{d S}{d T}=\frac{T}{4} \frac{d A_{H}}{d T}$, where $E$ is the energy, $S=\frac{A_{H}}{4}$ is the Bekenstein-Hawking entropy, and the first law $d E=T d S$ was used. We see that $C>0$, so the solution is locally stable. Even if $\beta$ as a function of $A_{H}$ is not monotonic, the part of $\beta(A)$ in the vicinity of $A_{B}$ is monotonically decreasing, and the above reasonings apply.

Statement 6. For sufficiently high temperature, a black hole phase is favorable both locally and globally.

Proof. It is quite obvious that for very high temperatures configuration with a black hole will dominate. Indeed, in the Eucldiean action approach, the free energy $F=T I$, the Euclidean action for a black hole topology $I=\beta E-S[4]$. When $\beta \rightarrow 0$, the first term in $I$ is negligible, so $I<0$. Meanwhile, for a hot empty space $I=0$. As a black hole exists for sufficiently high temperature $T>T_{m}$ and becomes thermodynamically favorable in the limit $T \rightarrow \infty$, there exists some $T_{1}>T_{m}$ such that for $T>T_{1}$ a black hole not only stable locally (see above) but also globally. Statements $4-6$ generalize the corresponding properties for Schwarzschild black holes [1].

\section{CONCLUSIONS}

Thus we showed that, defining boundary data, the canonical ensemble does exist if a temperature is high enough. Moreover, inside a cavity a black hole is favorable thermodynamically for sufficiently big temperature. This is done in a quite general approach without appealing to the explicit form of solutions that, generically, cannot be found at all. In doing so, the full system of Einstein equations was not used directly. We relied only on one relation (5), and the expression for the Kretschmann scalar (7) for vacuum space-times (in which, though, the validity of Einstein equations was already taken into account indirectly). We also proved rigorously that for vacuum black holes the Hawking temperature diverges when 
the horizon area shrinks. It would be of interest to check discussed properties for nonvacuum backgrounds and apply them to nucleation of black holes in cosmological problems.

\section{Acknowledgments}

This work was funded by the subsidy allocated to Kazan Federal University for the state assignment in the sphere of scientific activities. O. Z. also thanks for support SFFR, Ukraine, Project No. 32367.

[1] York, J.W., Jr.: Black-hole thermodynamics and the Euclidean Einstein action, Phys. Rev. D 33, 2092 - 2099 (1986)

[2] Gross, D. J., Perry M. J., and Yaffe L. G.: Instability of flat space at finite temperature. Phys. Rev. D 25, 330 - 355 (1982)

[3] Martinez, E. A. and York, J.W., Jr.: Additivity of the entropies of black Holes and matter, Phys. Rev. D 40, 2124 - 2127 (1989)

[4] Zslavskii, O. B.: Canonical ensemble for arbitrary configurations of self-gravitating systems, Phys. Lett. A 152, 463-466 (1991).

[5] Whiting, B. F. and York, J. W., Jr. Action Principle and Partition Function for the Gravitational Field in Black-Hole Topologies, Phys. Rev. Lett. 61, 1336 - 1339 (1988).

[6] Brown, J. D. , Comer, G. L. Martinez, E. A., Melmed, J., Whiting, B. F. and York, J. W., Jr.: Thermodynamic ensembles and gravitation, Class. Quant. Grav. 7, 1433 - 1444 (1990)

[7] Braden, H. W., Brown, J. D., Whiting, B. F., and York J. W., Jr.: Charged black hole in a grand canonical ensemble, Phys. Rev. D 42, 3376 - 3385 (1990)

[8] Peça, C. and Lemos, J. P. S.: Thermodynamics of Reissner-Nordstrom-anti-de Sitter black holes in the grand canonical ensemble, Phys. Rev. D 59, 124007 (1999) arXiv:gr-qc/9805004

[9] Israel, W. Event horizons in static vacuum space-times. Phys. Rev. 164, 1776 - 1779 (1967).

[10] Medved, A. J. M., Martin, D. and Visser, M.: Dirty black holes: Spacetime geometry and near-horizon symmetries, Class. Quantum Grav. 21, 3111-3125 (2004) arXiv:gr-qc/0402069 